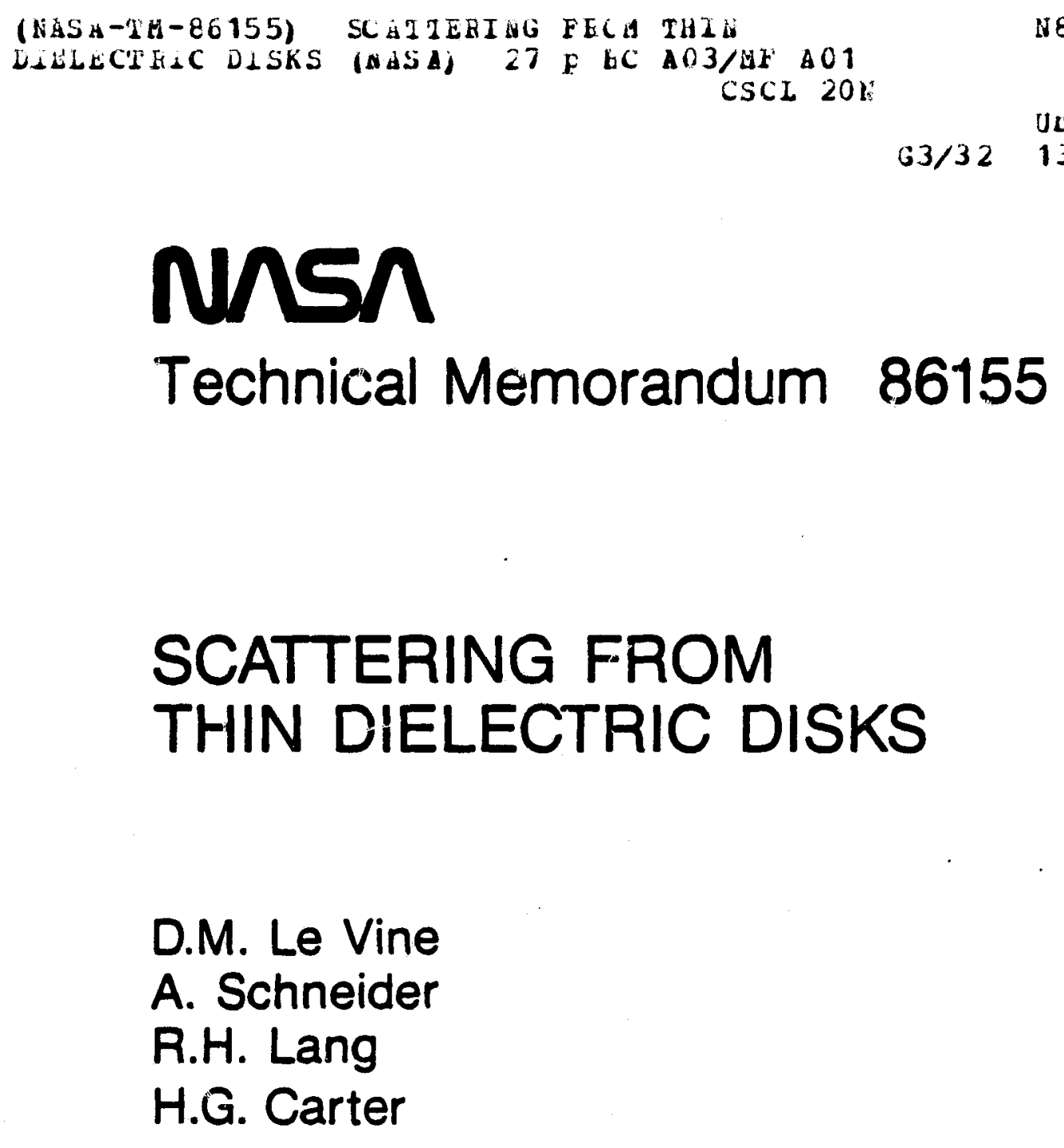

D.M. Le Vine
A. Schneider
R.H. Lang
H.G. Carter

September 1984

National Aeronautics and

Space Administration

Goddard Space Flight Center

Greenbelt, Maryland 20771 


\title{
SCATTERING FROM THIN DIELECTRIC DISKS
}

by

\author{
D.M. Le Vine \\ Goddard Laboratory for Atmospheric Sciences \\ Goddard Space Flight Center \\ Greenbelt, Maryland
}

\author{
A. Schneider \\ CyberCom Corporation \\ Arlington, Virginia \\ R.H. Lang \\ Department of Electrical Engineering \& Computer Science \\ Genrge Washington University \\ Washington, D.C. \\ H.G. Carter \\ Microwave Materials Laboratory \\ General Dynamicis Corporation \\ Fort Worth, Texas
}




\title{
SCATTERING FROM THIN DIELECTRIC DISKS
}

\author{
D.M. Le Vine \\ Goddard Laboratory For Atmospheric Sciences \\ Goddard Space Flight Center \\ Greenbelt, Maryland \\ A. Schneider \\ CyberCom Corporation \\ Arlington, Virginia \\ R.H. Lang \\ Department of Electrical Engineering \& Computer Science \\ George Washington University \\ Washington, D.C. \\ H.G. Carter \\ Microwave Materials Laboratory \\ General Dynamics Corporation \\ Eort Worth, Texas
}

A solution has been obtained for $s$. .ring from thin dielectric disks by approximating the currents induced inside the disk with the currents which would exist inside a dielectric slab of the same thickness, orientation and dielectric properties. This approximation reduces to an electrostatic approximation when the disk thickness, $T$, is small compared to the wavelength of the incident radiation and the approximation yields a conventional physical optics solution when the dimension, A, characteristic of the geometrical cross section of the disk (e.g. the diameter of a circular disk) is large compared to wavelength. When the ratio $A / T$ is sufficiently large the disk will always be in one or the other of these regimes $(T<<\lambda$ or $k A>>1)$. Consequently, when $A / T$ is large this solution provides a conventional approximation for the scattered fields which can be applied at all frequencies. As a check on this conclusion, a comparison has bsen made between the theoretical and measured radar cross section of thin dielectric disks. Agreement was found for thin disks with both large and small values of $\mathrm{kA}$.

$$
\begin{aligned}
& \text { PRTCEDTYC PAGE ELANK NOT FLMTED } \\
& \text { - iii }
\end{aligned}
$$




\section{INTRODUCTION}

Dielectric disks are important as models for objects encountered in nature such as the leaves of trees or crops and the ice crystals formed in clouds at high altitude (Sheppard and Holt. 1983). Unlike the case of the perfectly conducting disk (Meixner and Andrejewski, 1950; Ruck et al., 1970; Bowinan et al., 1969) an exact solution for scattering from a dielectric disk has not yet been found. Approximations have been reported which apply in the high and low frequency extremes (Ruck ex al., 1970; Bowman et al., 1969; Schiffer and Thielhiem, 1981) and numerical procedures have been developed for the intermediate regime (Weil and Chu, 1976a.b: Sheppard and Hclt, 1983); however. a single convenient approximation which can be used for all frequencies does not exist. The purpose of this article is to present an approximation for the electromagnetic fields scattered from dielectric disks which can be expressed in closed form and which applies for all frequencies when the disk is very thin. (The term "disk" is being used here to denote an object formed by cutting a thin slice from a cylinder of arbitrary cross section. The symbol " $\mathrm{T}$ " will denote the thickness of the slice and "A" will denote a length characteristic of the cross section of the cyliader, such as the radius if the cross section is circular. A thin disk is one for which $\mathrm{A} / \mathrm{T}>>1$.)

The approach is based on a solution for scattering from dielectric disks developed by Le Vine et al. (1983) which applies in the high frequency limit $(k A>>1)$. This solution was obtained by approximating the fields inside the disk b: the fields which would exist inside a slab of the same thickness, orientation and dielectric as the disk. This approximation ignores edge effects but has the advantage of using a solution to Maxwell's equations to approximate the fields inside the disk. As a result it applies regardless of the thickness and dielectric properties of the disk. It will be shown in this paper that for disks which are thin compared to wavelength $(T<<\lambda)$, this solution is also identical to an electrostatic approximation. Sufficiently thin disks $(A / T>>1)$ will be in one or the other of these regimes (i.e. $\Gamma<<\lambda$ or $k A>>$ ) depending on the frequency of the incident radiation. Consequently, in this case, this one solution will approximate scattering from the disk at 
all frequencies (all values of $k A$ ), either as an electrostatic approximation when $T<<\lambda$ or as a physical optics approximation at higher frequencies when $T$ is not small compared to wavelength. This later conclusion has been tested by comparing the theory with measurements. Comparisons will be presented to show that the theory agrees with measurements of scattering from thin dielectric disks boch when $k A>>1$ and 3 iso when $k A \cong 1$.

In the sections to follow an outline will be presented first of the theory for the scattered electric field, then the equivalence of this theory with the electrostatic approximation will be demonstrated for thin disks, and finally compatison with measurements will be made to verify that this single theory can apply for both large and small $\mathrm{kA}$. 


\section{SOLUTION}

Consider a plane wave incident on an arbitrarily oriented dielectric disk. The plancs wave is assumed to have polarization (direction of the electric field) $\hat{q}$ and to be propagating in the $\hat{i}$ direction:

$$
\bar{E}_{i n c}(\bar{t})=q E_{0} e^{j k_{0} i \cdot \bar{t}}
$$

where $k_{0}=\omega \sqrt{\mu_{0} \epsilon_{0}}$ and $E_{0}$ is the amplitude of the plane wave. The disk is assumed to have cross sectional shape $S(\bar{r})$, where $S(\bar{r})=1$ on the disk, and $S(\bar{r})=0$ otherwise, and to be comprised of material with relative dielectric constant $\epsilon_{p}=\epsilon_{p}^{\prime}+j \epsilon_{p}^{\prime \prime}$. It is assumed that $\epsilon_{p}$ is constant throughout the disk and that the share of the disk $S(\bar{r})$ is arbitrary. It is desired to determine the electric field, $\overline{\mathrm{E}}_{\text {scal }}(\overline{\mathrm{r}})$, scattered by the disk.

A formal solution for the scamered fieids can be obtained in terms of the fields inside the disk using standard procedures (e.g Ruck, et al., 1970; Le Vine et al., 1983). One obtains:

$$
\overline{\mathrm{E}}_{\text {scat }}(\overline{\mathrm{r}})=k_{0}{ }^{2} \int_{\text {volume }}\left(\epsilon_{\mathrm{r}}-1\right) \overline{\mathrm{E}}\left(\overline{\mathrm{r}}^{\prime}\right) \cdot \overline{\overline{\mathrm{G}}}\left(\bar{r} / \bar{r}^{\prime}\right) d \bar{r}^{\prime}
$$

where $\overline{\bar{G}}\left(\bar{r} / \bar{r}^{\prime}\right)$ is the dyadic Green's function for free space: $\overline{\bar{G}}\left(\bar{r} / \bar{r}^{\prime}\right)=\left(\overline{\bar{I}}+\frac{1}{k_{0}{ }^{2}} \bar{\nabla} \bar{\nabla}\right) \frac{e^{j k_{0} R}}{4 \pi R}$ and $R=\left|\bar{r}-\bar{r}^{\prime}\right|$ is the distance between the source point $\bar{r}^{\prime}$ and the observation point $\bar{r}, A$ solution will be sought in the far field of the disk $k_{0} R>>1$ ) in which case

$$
\overline{\bar{G}}\left(\bar{r} / \bar{r}^{\prime}\right) \equiv(\overline{\bar{I}}-\hat{\phi} \hat{0}) \frac{e j k_{0}\left(R_{0}-\hat{o} \cdot \bar{r}^{\prime}\right)}{4 \pi R_{0}}
$$

where $R_{0}$ is the distance from the conter of the disk to the observer and $o$ is a unit vector from the coordinate origin at the center of the disk toward the observer. To obtain a solution with this formalism the fields inside the disk $\bar{E}(\bar{r})$ must be obtained. These are not known in general and will be 
approximated here with the fields inside an identically oriented slab with the same dielectric properties and thickness as the disk. This approximation is similar to the Kirchhoff approximation employed in physical optics to solve for scattering from perfectly conducting surfaces. It ought to be reasonable when edge effects are not important such as in the case of disks whose cross section is much greater than a wavelength. This approximation has the advantage that it employs a canonical form (i.e., the fields inside the disk are a solution to Maxwell's equations) which also is the correct solution in a limiting case (a disk with infinite cross section).

The fields inside the slab are obtained in the usual manner by assuming plane wave modes inside and outside of the slab and matching boundary conditions at the slab surfaces (e.g. Born and Wolf. 1959). The procedure is most conveniently executed by resolving the incident wave into components polarized horizontally (perpendicular polarization) and vertically (parallel polarization) with respect to the plane defined by $\hat{\imath}$ and the normal to the disk. $\hat{n}$. The details for disks of arbitrary orientation are described in Le Vine et al (1983). For simplicity letting $\hat{\mathbf{n}}=\hat{z}$, which is suitable for purpoites of the discussion here, the result can be written:

$$
\begin{aligned}
\overline{\mathrm{E}}_{\mathrm{slab}}(\bar{r}, \hat{q})= & \left(\hat{q}_{\epsilon}^{+} e_{q}^{+} \exp \left(j k_{\alpha} \beta_{z} z\right)+\right. \\
& \left.+\hat{q}_{\epsilon}^{-} e_{q}^{-} \exp \left(-j k_{\alpha} \beta_{z} z\right)\right\} \exp \left(j k_{j} \bar{\beta}_{i} \cdot \bar{r}_{l}\right)
\end{aligned}
$$

In Equation 3 the vectors $\bar{\beta}^{ \pm}=\bar{\beta}_{1} \pm \beta_{z} \bar{z}$ indicate the direction of propagation of the two modes inside the slab and the unit vectors $\hat{q}_{\epsilon}^{ \pm}$denote the direction of polarization of these two waves: $\hat{\mathrm{q}} \frac{ \pm}{\epsilon}=\hat{h} \frac{t}{\epsilon}$ when the incident wave is horizontally polarized $(q=h)$ and $\hat{q} \frac{ \pm}{\epsilon}=\hat{v} \frac{ \pm}{\epsilon}$ when the incident wave is vertically polarized $(q=v)$ as defined in figure 1 . Also the propagation vectors $\bar{\beta} \pm$ have been resolved into components parallel and perpendicular to the disk (i.e. $\bar{\beta}^{ \pm}=\bar{\beta}_{1}+\beta_{z}$ ), and orie can show (Le Vine et al., 1983) that $\beta_{z}=\bar{\beta}^{+} \cdot \hat{n}=\sqrt{\epsilon_{\mathrm{r}}-1+(\hat{\mathbf{i}} \cdot \hat{\mathbf{n}})^{2}}$.. The scalars, $\mathrm{e} \frac{t}{\mathrm{q}}$, in Equation 3 are characteristics of the slab obtained by marching boundary conditions at the slab interfaces. When a coordinate system is chosen with the origin at the center of the slab (i.e. halfway between the (wo faces), these scalars are: 


$$
\begin{aligned}
& e_{q}^{+}=-\frac{t_{q} r_{q} e^{j \downarrow}}{1-r_{q}{ }^{2} e^{j \downarrow}} \exp \left[j / 2 k_{o} T(\hat{i}+\bar{\beta}-) \cdot \hat{n}\right], \\
& e_{q}^{-}=\frac{t_{q}}{1-r_{q}{ }^{2} e^{j \downarrow}} \exp \left[j / 2 k_{0} T\left(\hat{i}+\bar{\beta}^{+}\right) \cdot \hat{n}\right],
\end{aligned}
$$

where $\psi=2 k_{0} T\left(\vec{\beta}^{\circ} \cdot \hat{n}\right)$ and

$$
\begin{aligned}
& r_{q}=\frac{(\hat{i} \cdot \hat{n})+\delta_{q}\left(\overline{\beta^{*}} \cdot \hat{n}\right)}{(\hat{i} \cdot \hat{n})-\delta_{q}\left(\bar{\beta}^{\bullet} \cdot \hat{n}\right)} \\
& t_{q}=\frac{+2 \sqrt{\delta_{q}}(\hat{i} \cdot \hat{n})}{(\hat{i} \cdot \hat{n})-\delta_{q}\left(\bar{\beta}^{\bullet} \cdot \hat{n}\right)} \\
& \delta_{q}= \begin{cases}1 & q=h \\
\frac{1}{\epsilon_{q}} & q=v\end{cases}
\end{aligned}
$$

Le Vine et al. (1983) have solved for the scattered field of polarization $\dot{p}$ due to an incident wave of polarization $\hat{q}$ in terms of a dyadic scattering amplitude $\overline{\bar{f}}(\hat{o}, \hat{i})$ :

$$
\hat{p} \cdot \bar{E}_{\operatorname{scas}}(\bar{r}, \hat{q})=[\hat{p} \cdot \overline{\bar{f}}(\hat{o}, \hat{i}) \cdot \hat{q}] E_{j} \frac{e^{j k_{0} R_{0}}}{R_{0}}
$$

and they have shown that when Equation 3 is substituted into Equation 2, one obtains

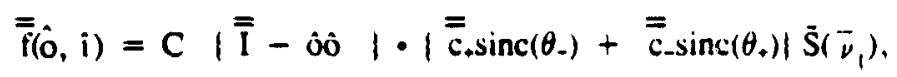

where

$$
\begin{aligned}
\overline{\bar{c}}_{ \pm} & =\hat{h} h e_{h} \pm+\frac{1}{\sqrt{\epsilon_{r}}}\left(\hat{v}+\bar{\Omega}_{ \pm}\right) \hat{v} e_{v}^{ \pm}, \\
\bar{\Omega}_{ \pm} & =\left[-\hat{i}+\bar{\beta}^{ \pm}\right] \cdot \hat{n}(\hat{\mathrm{n}} \times \hat{n}), \\
\theta \pm & = \pm \frac{1}{2} \mathrm{k}_{0} T[\hat{0}+\bar{\beta} \pm] \cdot \hat{n} \\
C & =\frac{T}{4 \pi} k_{0}^{2}\left(\epsilon_{r}-1\right),
\end{aligned}
$$


In these expressions $S\left(\vec{v}_{t}\right)$ is the Fourier transform of $S(\bar{r})$ with resnect to the cartesian coordinates, $\bar{r}_{f}$ in the plane perpendicular to the normal to the disk; and the unit vectors $\hat{h}$ and $\hat{v}$ denote the direction of horizontal a vertical polarization of the incident wave (as shown in Figure 1).

As a check on this solution, Le Vine (1984) has compared the radar cross section obtained using Equation 7 with the radar cross section reported in the literature for special cases and obtained using other approaches. The radar cross section can be obcained from Equation 7 using the definition

$$
\sigma_{p q}=4 \pi|\hat{p} \cdot \hat{\bar{f}}(-\hat{i}, \hat{i}) \cdot \hat{q}|^{2}
$$

and substituting Equation 7 into Equation 9 resul ${ }^{-\cdots}$ in the following simple expression (Le Vine, 1984):

$$
\sigma_{p q}=\sigma_{\infty}\left|R_{p}\right|=\delta_{p q}
$$

where $\sigma_{\infty}$ is the physical optics form obtained for the backscatter cross section of a perfectly ing disk of the same shape, $R_{p}$ is the Fresnel reflection coefficient for a wave of polarization $\hat{p} \in\{\hat{h}, \hat{v}\}$ incident on an identically oriented slab of the same thickness and dielectric properties as the disk, and $\delta_{p q}=0$ if $p \neq q$ and $\delta_{p q}=1$ if $\hat{p}=\hat{q}$. Equation 10 agrees with special cases previously obtained using other approaches: It reduces to the physical optics solution for scattering from perfectly conducting disks when the disk is made very lossy; it reduces to the form obtained by Schiffer and Thielhiem (1981) for very thin disks; and at normal incidence, it has the form obtained by Neugebauer (1956, 1957) and verified by experiment (Ruck et al., 1970).

It is also interesting to observe that Equation 7 reduces to the result obtained with a Born approximation when the relative dielectric constant is not large (e.g. $\epsilon_{\mathrm{r}} \cong 1$ ) and the change in phase as the wave propagates through the disk is small $\left[k_{0} T\left(\bar{\beta}^{+} \cdot \hat{n}\right)<<1\right]$. In this case $\bar{\beta}^{+} \cdot \hat{n} \equiv-\hat{i} \bullet \hat{n}$ because $\epsilon_{\mathrm{r}} \cong 1$. Consequently, $\mathrm{r}_{\mathrm{q}} \cong 0, \mathrm{t}_{\mathrm{q}} \cong 1$ and $\mathrm{e}_{\mathrm{q}}^{+} \equiv 0, \mathrm{e}_{\mathrm{q}}^{-} \cong 1$. Using these results in Equation 3 it is found that the wave inside the slab is approximately the incident wave, just as is assumed in the Born approximation (Ishimaru, 1978; Bohren and Huffman, 1983). 


\section{EQUIVALENCE WITH THE ELECTROSTATIC APPROXIMATION}

In the limit of very thin disks the fields inside the disk (Equation 3) reduce to those oblained assuming an incident electrostatic field at the slab boundary with the same direction (polarization) and phase as the electric field associated with the incident plane wave. When $k A<<1$ the change in phase across the disk is neglible making the use of the electrostatic approximation in Equation 2 equivalent to Rayleigh scattering (Ishimaru, 1980; Bowman et al., 1980).

To see that Equation 3 reduces to the electrostatic approximation for thin disks notice that when $k_{0} T$ is smail, $\exp \left( \pm j k_{0} \beta_{2} z l \equiv 1\right.$ and $\exp \left(j k_{0} T\right) \equiv 1$. Hence, Equation 3 becomes

$$
\overline{\mathrm{E}}_{\mathrm{slab}}(\overline{\mathrm{r}}, \hat{\mathrm{q}})=\left[\hat{\mathrm{q}}_{\mathrm{t}}^{+} \mathrm{e}_{\mathrm{q}}^{+}+\hat{\mathrm{q}}_{\epsilon}^{-} \bar{\epsilon}_{\mathrm{q}}^{-}\right] \exp \left(\mathrm{jk} \mathrm{k}_{0} \bar{\beta}_{\mathrm{t}} \cdot \overline{\mathrm{r}}_{\mathrm{t}}\right)
$$

and

$$
\begin{aligned}
& e_{q}^{+}=\frac{t_{1} r_{q}}{1-r_{q}^{2}} \\
& e_{q}^{-}=\frac{t_{q}}{1 r_{q}^{2}}
\end{aligned}
$$

where $q \in\{h, v\}$. As evident from Equation 11 , when $k_{j} T$ is small the fields inside the disk are constants with respect to $z$ and vary only with the coordinate, , $\bar{r}_{i}$, parallel to the surface of the disk. The constant is given by the terms in brackets on the right in Equation 11. It has the value obtained in statics when a unit amplitude electric field with direction (polarization) $\hat{q}$ is applied to the slab.

To see this, consider separately the cases when the incident wave is horizontally or vertically polarized. When the incident wave is horizontally polarized $(q=h)$, the polarization vectors of the waves inside the slab are $\hat{h}_{\epsilon}^{\ddagger}$ (see Figure 1). Hence, the term in brackets in Equation 11 is:

$$
\begin{aligned}
\hat{q}_{\epsilon}^{+} e_{q}^{+}+\hat{q}_{t}^{-} e_{q}^{-} & =\hat{h}_{\epsilon}^{+} e_{h}^{+}+\hat{h}_{\epsilon}^{-} e_{h}^{-} \\
& =\left[e_{h}^{+}+e_{h}^{-}\right] \hat{h} \\
& =\hat{h}
\end{aligned}
$$

The last step follows because $e_{h}+e_{\bar{h}}=1$ which is obtained from Equations 5 and 12 . Equation 13 
states that when the incident electric field is parallel to the boundary (it has unit amplitur': and direction $\hat{h}$ ), then the total electric field inside the slab and parallel to the boundary (i.e. in the directions $\hat{h}_{\epsilon}^{+}$and $\hat{h}_{-}^{-}$) has the same amplitude as the incident wave. That is, the tangential electric fields are continuous across the boundary. When the incident field is vertically polarized $(q=v)$ the electric fields inside the slab point in the $\hat{v}_{e}^{ \pm}$directions (Figure 1 ). In this case:

$$
\dot{q}_{e}^{+} e_{q}^{+i}+\dot{q}_{\epsilon}^{-} e_{q}^{-}=\dot{v}_{\varepsilon}^{+} e_{v}^{+}+\hat{v}_{\epsilon}^{-} e_{v}^{-}
$$

and resolving $\hat{v} \neq$ into components perpendicular and parallel to the disk one has:

$$
\begin{aligned}
\cdot \hat{v}_{e}^{+} e_{v}^{+}+\hat{v}_{e}^{-} e_{v}^{-}= & {\left[e_{v}^{+}+e_{v}^{-}\right] \sin \theta_{e} \hat{n}+} \\
& +\left(e_{v}^{+}-e_{v}^{-}\right] \cos \theta_{e}(\hat{n} \times \hat{h})
\end{aligned}
$$

where $\cos \theta_{i}=-\hat{i} \cdot \hat{n}$ and $\cos \theta_{\epsilon}=\bar{\beta}^{+}$. $\hat{n}$ are the angles the incident wave and waves inside the slab, respectively, make with the normal to the slab (Figure 1 ). The unit vector $\hat{n} \times \hat{h}$ is tangential to the surface of the disk and has the direction of the component of vertically polarized incident electric field which is tangential to the surface. Using the result $e_{v}^{+}-e_{v}^{-}=\cos \theta_{i} / \cos \theta_{\epsilon}$ (Equations 5 and 12) it is clear that this component has magnicude $\cos \theta_{i}$. Since this is also the magnitude of the component of vertically polarized incident field which is parallel to the boundary, this term is again a statement that the tangential component of the incident electric field equals the total tangential component of the electric field inside the slab. The remaining term in Equation $14 \mathrm{~b}$ is the component of the vertically polarized electric field normal to the boundary. It must satisfy the constraint that the normal compenent of electric field density (i.e. $\vec{D} \cdot \hat{\mathbf{n}}=\epsilon \overline{\mathrm{E}} \cdot \hat{\mathbf{n}}$ ) is continuous across the boundary. Equating the external applied component of $\bar{D}$ normal to the surface (of magnitude $\sin \theta_{i}$ ) with the total normal component inside the slab and using the relationship $e_{v}^{+}+e_{v}^{-}=1 / \sqrt{\epsilon_{p}}$ one obtains

$$
\frac{\sin \theta_{\epsilon}}{\sin \theta_{i}}=\frac{\eta_{\epsilon}}{\eta_{i}}
$$

where $\eta_{t}=\sqrt{\epsilon_{r}}$ is the index of refraction of the medium inside the disk and $\eta_{i}=1$ is the index of refraction of the medium outside the disk. Equation 15 is just Snell's law and determines $\theta_{\epsilon}$ in terms of $\epsilon_{\mathrm{r}}$ and $\theta_{\mathrm{i}}$. 


\section{COMPARISON WITH EXPERIMENT}

It has just been shown that when $k_{0} T<<1$ Equation 3 is equivalent to the solution obtained in statics for the fiektas inside the slab when a unit amplitude electric field is applied to the slab, except that at each point the solution is assigned the phase $\exp \left(j \mathrm{k}_{0} \bar{\beta}_{1} \bullet \bar{p}_{\mathrm{t}}\right)$ of the incident plane wave. When this result is used in Equation 2 for the fields inside the disk, it leads to an approximation sometimes called an electrostatic approximation (Bohren and Huffman, 1983.) When in addition, $k_{0} A<<1$ this solution is the conventional Rayleigh approximation (e.g. Ishimaru, 1980; Bowman et al., 1969). This follows because when $k_{0} A<<1$ the change in phase across the disk due to the incident plane wave is neglible.

In the other extreme, witen $k_{0} A>>1$, the use of Equation 3 in Equation 2 leads to a solution with properties similar to those obtained with the Kirchhoff approximation used in physical optics. The properties of the solution for the scattered fields in this case have been described by Le Vine et al (1983). In this case the solution applies ev"n when $k_{o} T>1$ as long as edge effects can be neglected. The solution in this regime has been shown to yield conventional results in special cases (Le Vine, 1984).

Equation 3 can be used to obtain approximations in such different frequency extremes because it is a correct solution to Maxwell's equations for a canonical problem, the slab. It correctly represents the fields inside the slab at all frequencies and for arbitrary slab parameters (e.g. thickness and dielectric constant). The intrinsic limitation on the use of Equation 3 as an approximation for the fields inside the disk is that it neglects the effects of the edges of the disk. These characteristics suggest that when edge effects are not important, the use of Equation 3 in Equation 2 :may accurately represent the scattered fields at all frequencies. Edge effects ought to be neglible for disks which are very thin compared to their physical cross section (i e. disks for which $\mathrm{A} / \mathrm{T}>>1$ ). 
An attempe has been maje to test this hypothesis by comparing the theory with measurements of scattering from thin dielectric disks. The literature on scattering from dielectric disks of any shape is sparse, and it is even more difficult to find measurements of scattering from dielectric disks for which A/T $>>$ I. Furthermore, in order to properly cest this theory daca is needed over the frequency range from $k A>>1$ to $k A<<1$. Measurements were found of scattering from dielectric disks with $A / T=$ 3,000 and $k A \geq 25$ which is quite suitable to test the theory in the high frequency regine ( $k A>>1$ ). The agreement between experiment and theory is quite good in this case. At the lower frequency regime $(k A<1)$ data was found for disks with $A / T \equiv 5$ and $k A \equiv 0.75$. The ratio $A / T$ is clearly smaller in this case than desirable to exclude edge effects; however, the measurements agree surprisingly well with theory for incidence angles within about $30^{\circ}$ of nadir. Details of these comparisons of theory and measurement are discussed below.

First consider the case when KA is large. A comparison has been made of the theoretical radar cross section obtained from Equations 7 and 9 with values of the radar cross section of very thin dielectric disks measured by H. G. Carter of the Microwave Materials Research Laboratory, General Dynamics Corporation, Fort Worth, Texas. These measurements (unpublished) were performed for J. Eckeran of System Planning Corporation (SPC-Technologies) who brought them to the author's atention. The experiment consisted of measuring the radar cross section of square sheets of nylon fabric. The fabric was mounted on a pedestal in an anechoic chamber and rotated so that the cross section could be measured as a function of incidence angle. The samples were $30.48 \mathrm{~cm}$ on a side and $.0106 \mathrm{~cm}$ thick $(\mathrm{A} / \mathrm{T} \equiv 3000)$ with a relative dielectric constant of $\epsilon_{\mathrm{r}}=2.272$. Measurements were made at frequencies of $16 \mathrm{GHz}, 9.75 \mathrm{GHz}$ and $4.0 \mathrm{GHz}(\mathrm{kA}=102,62$, and 26, respectively). Examples of the measured radar cross section $\sigma_{\text {hh }}$ at $16 \mathrm{GHz}$ and $9.75 \mathrm{GHz}$ are shown in Figures 2 and 3 and an example of $\sigma_{v v}$ measured at $4.0 \mathrm{GHz}$ is shown in Figure 4. Ideally, the measured crosis section should be symmetric about nadir. Asymmetries are apparent in the data and are most likely due to the strucrure holding the sample (a styrofoam box) or variations in the nulling procedure used to eliminate contributions from the background in this CW system (H. Carter, private communication). 
The noise level is about $-45 \mathrm{db}$ as is evident from the figures.

The theoretical radar cross section is obtained by substituting Equation 7 into Equation 9. For very thin disks the results simplify considerably (Le Vine, 1984) and one obtains:

$$
\begin{aligned}
& \sigma_{h h}=\left[\frac{\pi k_{0}^{2}\left(\epsilon_{r}-1\right)}{\sqrt{4 \pi}} X_{0}^{2} \operatorname{sinc}\left(k_{0} X_{0} \sin \theta\right)\right]^{2} \\
& \sigma_{v v}=\left[\frac{\sin ^{2} \theta-\epsilon_{r} \cos ^{2} \theta}{\epsilon_{r}}\right]_{h h}^{2}
\end{aligned}
$$

where $X_{0}$ is the size of one side of the square sample $\left(X_{0}=30.48 \mathrm{~cm}\right)$. The radar cross section obtained from Equations $16 \mathrm{ka}$ been plotted for comparison on the left in Figures 2-4. Comparing the pairs in each figure indicates good agreement between theory and mensurement. The agreement is very good near nadir. At angles off nadir the location of the nulls predicted by the theory are in good agreement with measurement and the envelope of the measured curves compares well with theory until the data becomes dominated by noise. The main differences between theory and measurement are asymmetries present in the data and not in the theory.

In the lnw frequency regime $(k A<1)$ comparison has been made with the measurements of Allan and vicCormick (1978; 1980). They measured scattering from dielectric disks of circular cross section and relative dielectric constant $\epsilon_{\mathrm{r}}=3.18+\mathrm{j} 0.036$. In these measurements the frequency of the radar was constant $(2.86 \mathrm{GHz})$ and measurements were made on several disks with different combinations of radius and thickness, yieiding a data set with $0.76 \leq \mathrm{kA} \leq 3.8$ and $1.25 \leq \mathrm{A} / \mathrm{T} \leq 5.0$. Measurements were made using circular polarization and the disks were rotated to obtain values of the rross section, $\sigma$, for circular prolarization as a function of incidence angle. Examples of $\sigma / \lambda^{2}$ (where $\lambda$ is the wavelength of the incident radiation) as measured by Allan and McCormick (1980) are plotted in Figures $5-7$ using the solid lines. Figure 5 shows $\sigma / \lambda^{2}$ for a disk with $A / T=5.0$ at $k A=0.76,2.3$ and 3.8. ( $\mathrm{A}$ is the radius of the disk). Figure 6 illustrates the scattering from several disks with the 
same $k A(k A=3.8)$ but with increasing thickness. The disks used for these measurements are shown to scale below each figure. Figure 7 shows the same data ploned as a function of disk thickness but at a smailer $k A(k A=1.5)$. Edge effects are clearly evident in all these data when the incidence angle is greater than about $60^{\circ}$. In fact, the thickest disks have cross sections which are almost as large at edge-on incidence $\left(90^{\circ}\right)$ as at nadir $\left(0^{\circ}\right)$.

The theoretical expression for the radar cross section fi.r circular polarization, $\sigma$, can be obtained from Equation 7 as follows:

$$
\begin{aligned}
\sigma & =\pi\left|f_{h h}+f_{v v}\right|^{2} \\
& =1 / 4\left[\sigma_{h h}+\sigma_{v v}+2 \pi \operatorname{Re}\left(f_{h h} f_{v v}^{*}\right)\right]
\end{aligned}
$$

where $f_{p q}=\hat{p} \cdot \overline{\bar{f}}(-\hat{i}, \hat{i}) \cdot \hat{q}$ and $\sigma_{h h}$ and $\sigma_{v v}$ are the radar cross sections for linear polarization as defined by Equation 9 . The theoretical values for the radar cross section. obcained by substituting Equation 7 into Equation 17 have been plotted as dashed curves in Figures 5-7. Equation 7 has been used without the simplification appropriate for thin disks used to obtain liquations 16 because in this data set $k_{0} T$ is not particularly small. Figure 5 shows a comparison of theory and measurement for the thinnest disk available in the data set $(A / T=5.0)$ at several values of $k A$. Notice that the agreement is good for incidence angles less than about $30^{\circ}$ at the larger values of $\mathrm{kA}$ and is reasonable even for $k A=0.76$. Figures 6 and 7 illustrate the importance of disk thickness on the agreement between theory and experiment. For the thinnest disk and the largest kA (Figure 6) the agreement between theory and measurement is good for incidence angles less than about $30^{\circ}$; however, for thicker disks the agreement deteriorates rapidly. One would expect the agreement to improve for disks with larger values of $\mathrm{A} / \mathrm{T}$ (e.g. $\mathrm{A} / \mathrm{T}>100$ ) for which the theory is more appropriate and a trend in this direction is evident. The theory ignores edge effects, as described above, and its failure to predict these effects is clear in the data (e.g. at incidence angles greater than $60^{\circ}$ ). However, for disks with much large $A / T$, for which the theory is intended, these effects ought to be significantly less in relative importance than in this data set. 


\section{v. CONCLUSIONS}

A solution has been presented for scattering from thin dielectric disks which applies for all frequencies regardless of the size of the disk compared to wavelength. The solution is equivalent to either an electrostatic approximation or physical optics approximation depending on the ratio of thickness compared to wavelength. The solution applies whenever the dimension, $A$, characteristic of the cross section of the disk, is much larger than the disk's thickness (A/T $>>1$ ). This is a restriction which is not unreasonable for many objects occuring in nature for which a disk might be used as a model: For example, $A / T=500$ for mature leaves of soybean plants.

In order to check that the theory does apply over a wide range of $\mathrm{kA}, \mathrm{a}$ comparison was made with available measurements of scattering from thin dielectric disks. The ideal experiment would consist of measuring the scatter from a single thin disk as function of frequency proceeding from $k A<<1$ through the resonant region to $k A>>1$. If the theory is valid, it should describe the scattered fields at each frequency regardless of $k A$ for disks for which $A / T>>1$. Although the ideal experiment was not performed, data were located on scattering from disks at large and small values of $k A$. The agreement between data and experiment was gond when $k A>>1$, apparently being limited in this case more by noise in the experiment than limitations in the theory. For the case when kA was not large, the size in thickness ratio of the disks used in the measurements was marginal for applicability of the theory $(A / T=5)$, and it would not have been surprising had the agreement been poior. However, the data showed surprisingly good agreement with the measurements at incidence angles less than $30^{\circ}$. The agreement seemed to improve with increases in $\mathrm{kA}$ and $\mathrm{A} / \mathrm{T}$ as expected from the theory. 


\section{ACKNOWLEDGEMENT:}

The authors would like to thank Jerome Eckerman for his interest in this work and for his assistance in bringing Dr. Carter's measurements to our attentinn. We also wish to acknowledge the cooperation of L. A.llan in the sharing of his data and S. S. Seker whose computer codes for scattering from disks were used in some of the numerical work. 


\section{REFERENCES}

Alian. L.E. and G.C. McCormick. (1978), "Measurements of the Backscatter Matrix of Dielectric Spheriods," IEEE Trans. on Antennas and Propagation, Vol. AP-26 ("44). pp. 579-587.

Allan, L. E. and G.C. McCormick, (1980), "Measurements of the Backscatter Matrix of Dielectric Bodies," IEEE Trans. on Antennas and Propagation, Vol. AP-28 (12), pp. 166-169.

Bohren, C.F. and D.R. Huffman, (1983), Absorption and Scattering of Light by Small Particles, Wiley, New York.

Born, M. and E. Wolf, (1959), Principles of Optics, Pergamon Press, Section 3.5.

Bowman, I.J., T.B.A. Senior and P.L.E. Uslengi, Editors, (1969), Electromagnetic and Acoustic Scattering by Simple Shapes, North-Holland, Chapter 14 (by F.B. Sleator)

Ishimaru, A. (1978), Wave Propagation and Scattering in Random Media, Academic Press, New York, Vol. I, Chapter 1.

Le Vine, D.M. and R. Meneghini, R.H. Lang and S.S. Seker, (1983), "Scattering from Arbitrarily Oriented Dielectric Disks in the Physical Optics Regime," J. Optical Society of America, Vol. 73 (\#10) pp. 1255-1262.

Le Vine, D.M., (1984). "The Radar Cross Section of Dielectric Disks," IEEE Trans. Antennas and Propagation, Vol. AP 32 (\#1), pp. 6-12.

J. Meixner and W. Andrejewski, "Strenge Theorie der Beugung ebener and elektromagnetischer Wellen an der volkommen leitenden Kreisscheibe und an der kreisformigen Offnung im leitenden ebenen Schirm," Ann. Phys., vol. 7, p. 157, 1950.

Ruck, G.T., D.E. Barrick, W.D. Stuart and C.K. Kirchbaum, (1970), Radar Cross Section Handbook. Vol, 2, Plenum Press, New York.

Schiffer, R. and K.O. Thielheim (1979), "Light Scattering by Dielectric Needles and Disks," J. Applied Physics, Vol. $50(\# 4)$, pp. 2476-2483. 
Weil, H. and C-M Chu, (1976a), "Scattering and Absorption of Electromagnetic Radiation by Thin Dielectric Disks." Applied Optics, Vol. 15 (\$7), pp. 1832-1836.

Weil. H. and C.M Chu. (1967b), "Integral Equation Method for Scattering and Absorption of Electromagnetic Radiation by Thin Lossy Dielectric Disks.” J. Computational Phys., Vol. 22 ("l), pp. $111-124$. 


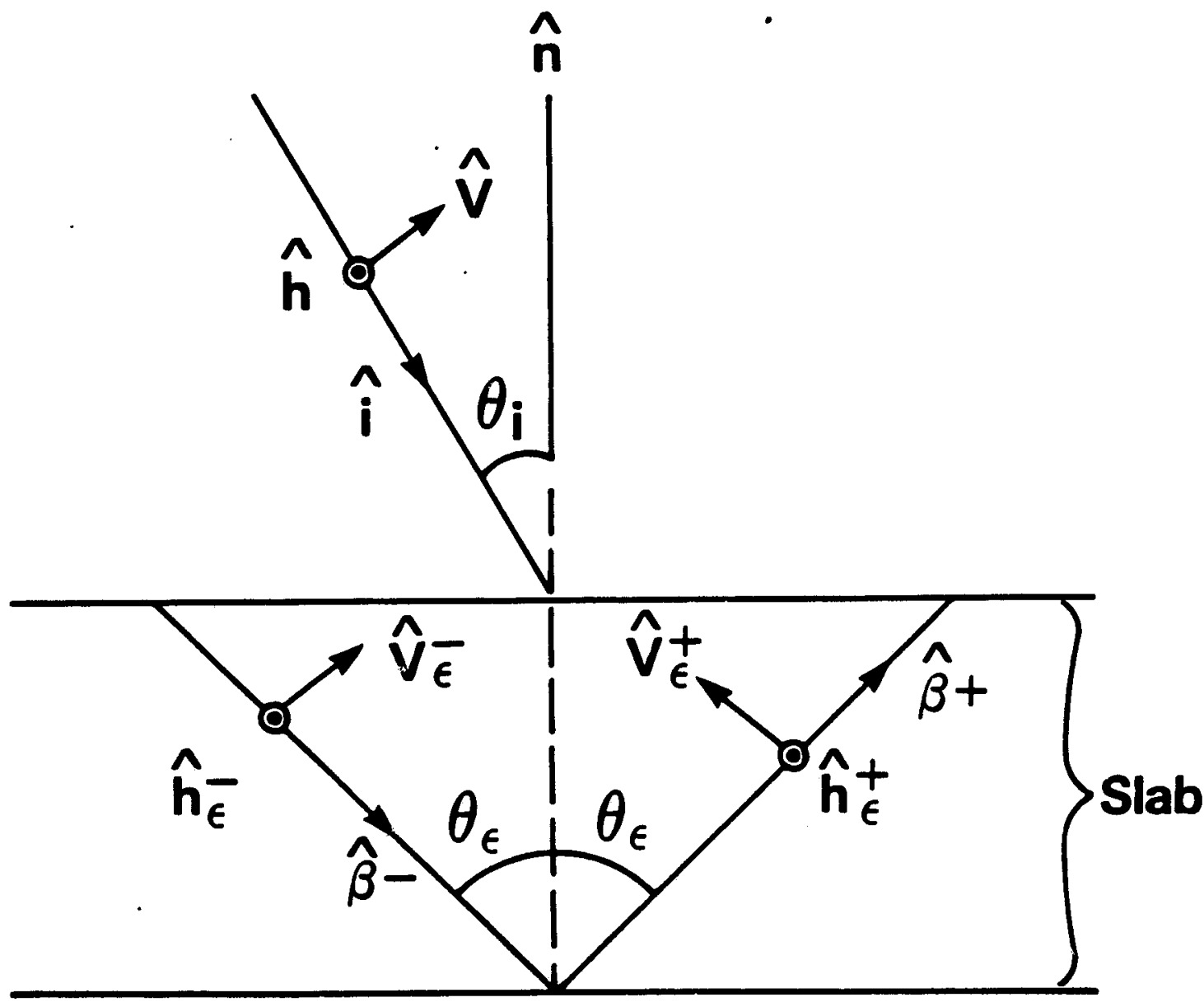

Figure 1. Polarization and propagation vectors for scattering from the slab. 


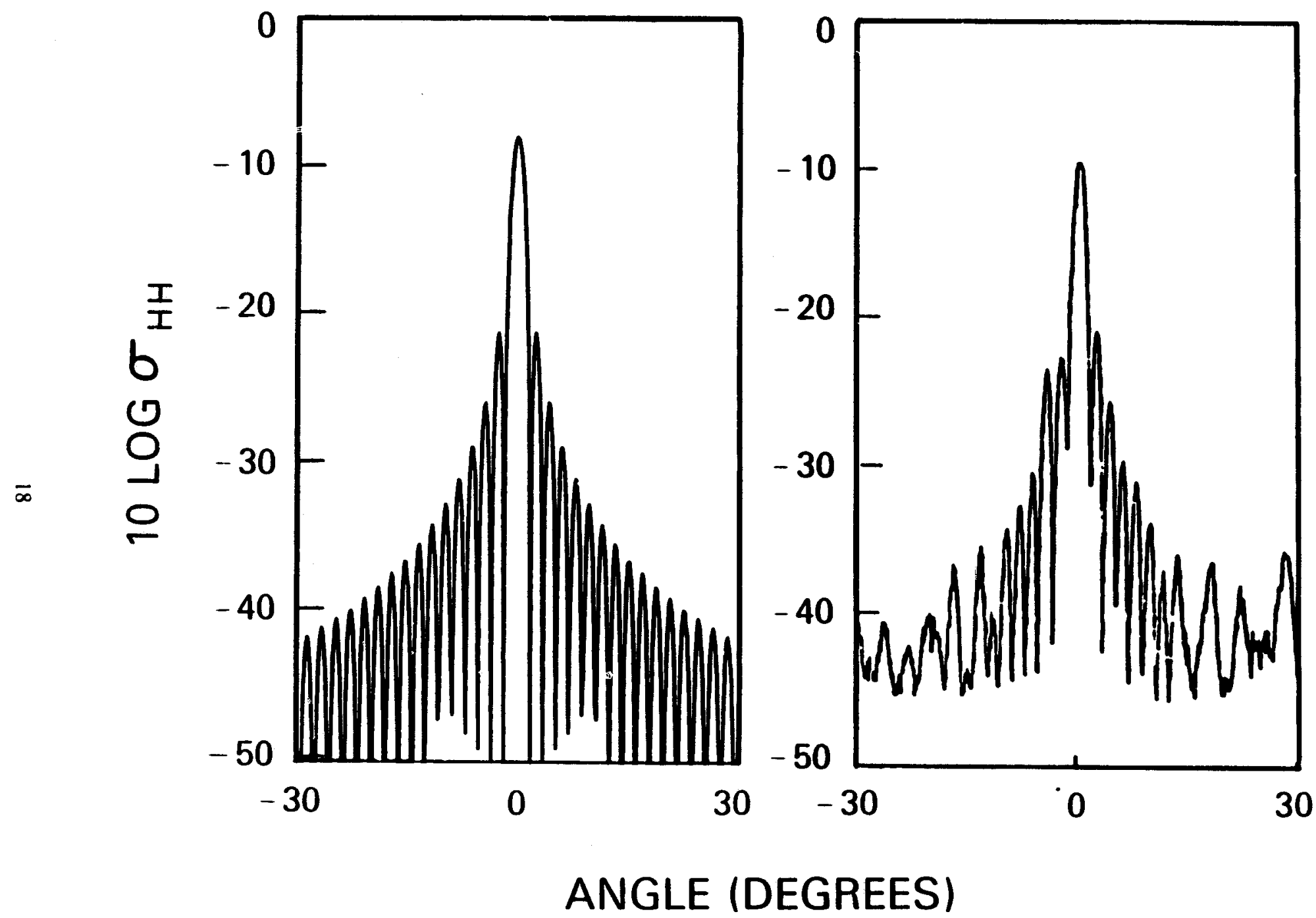

Figure 2. Scattering from a thin rectangular disk at $f=16 \mathrm{GHz}$. Theory (left) and measurements (right). 


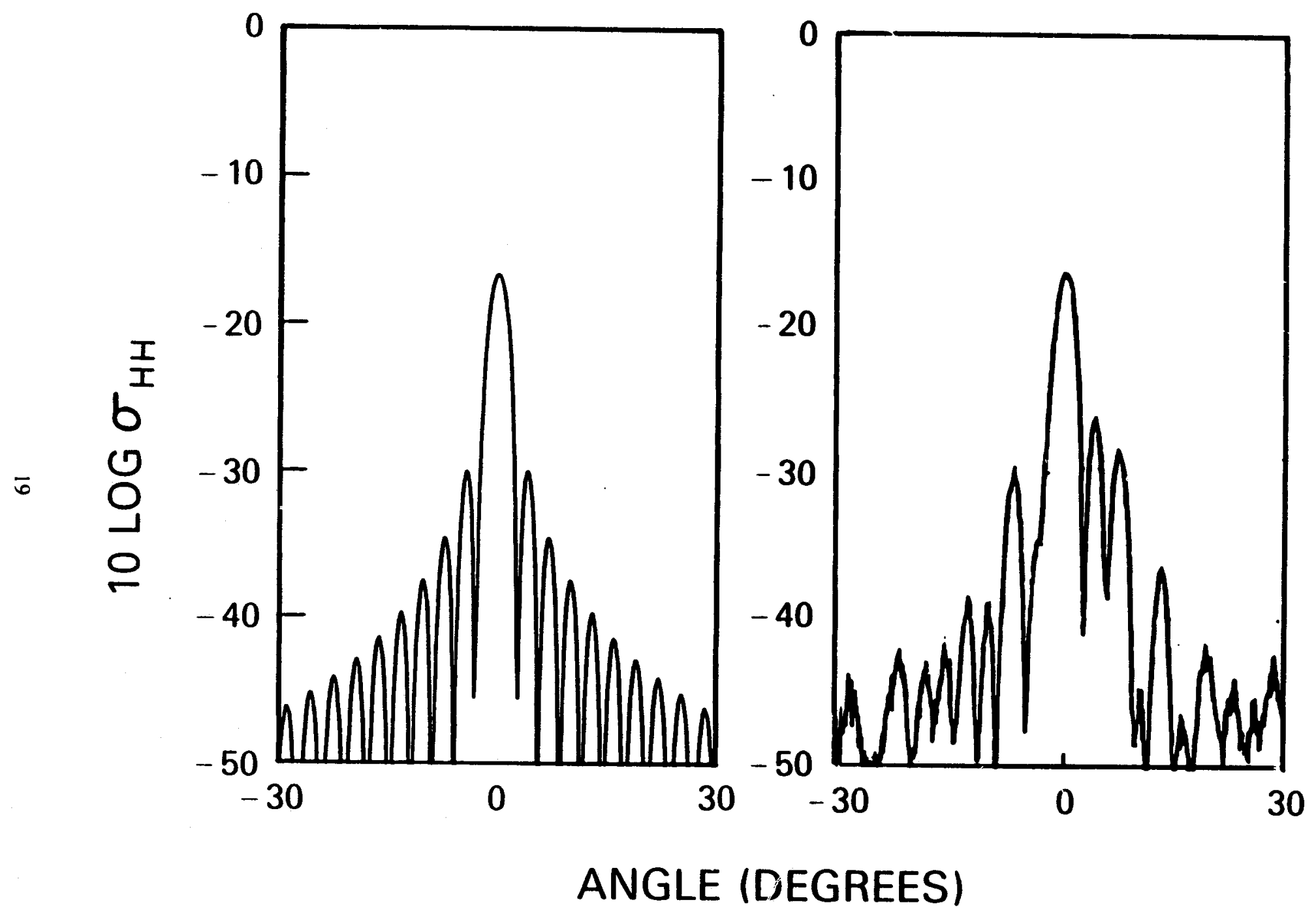

Figure 3. Scattering from a thin rectangular disk at $\mathrm{f}=9.75 \mathrm{GHz}$. Theory (lefi) and data (right), 


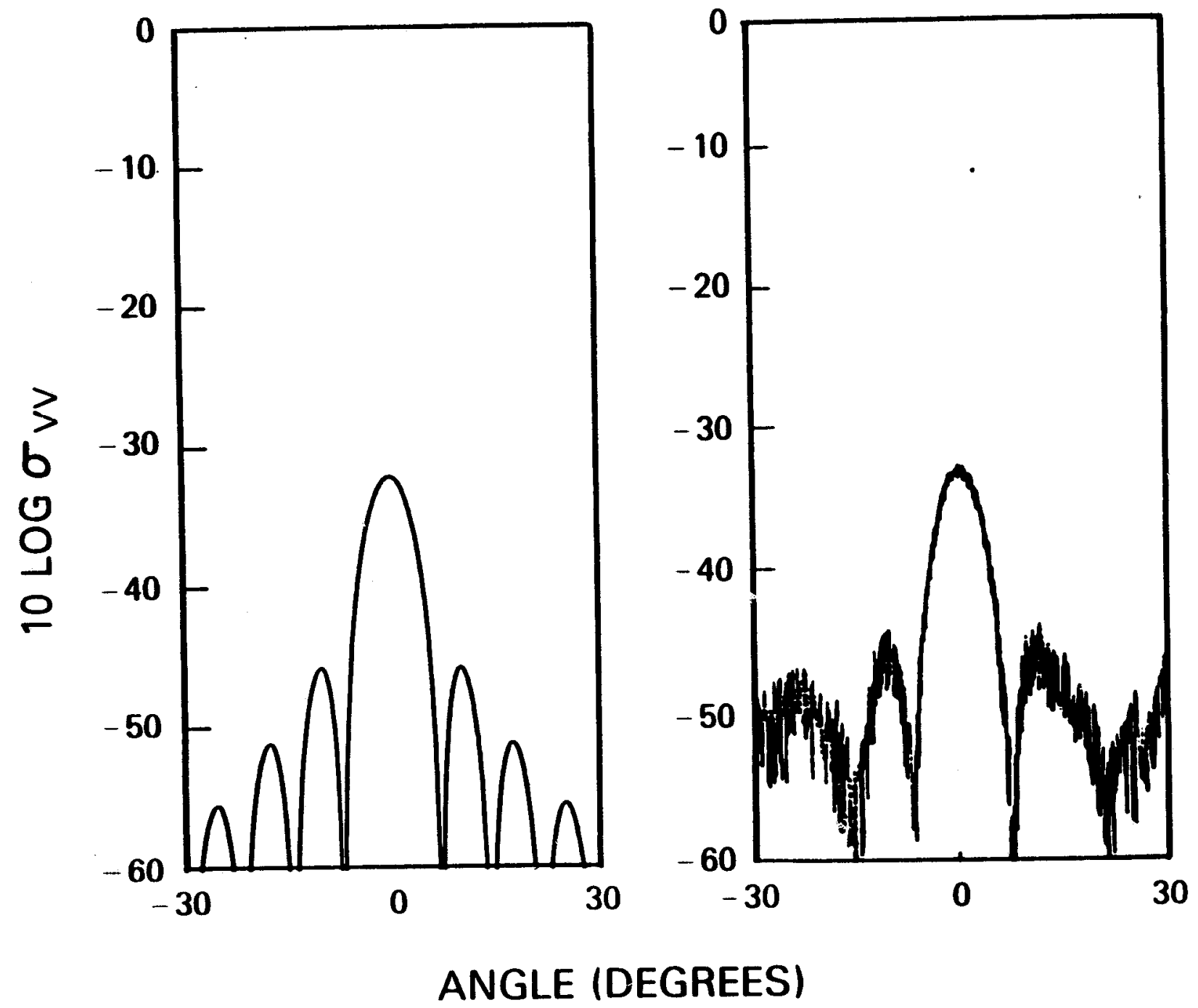

Figure 4. Scattering from a thin rectangular disk at $f=4 \mathrm{GHz}$. Theory (left) and measurements (righit). 

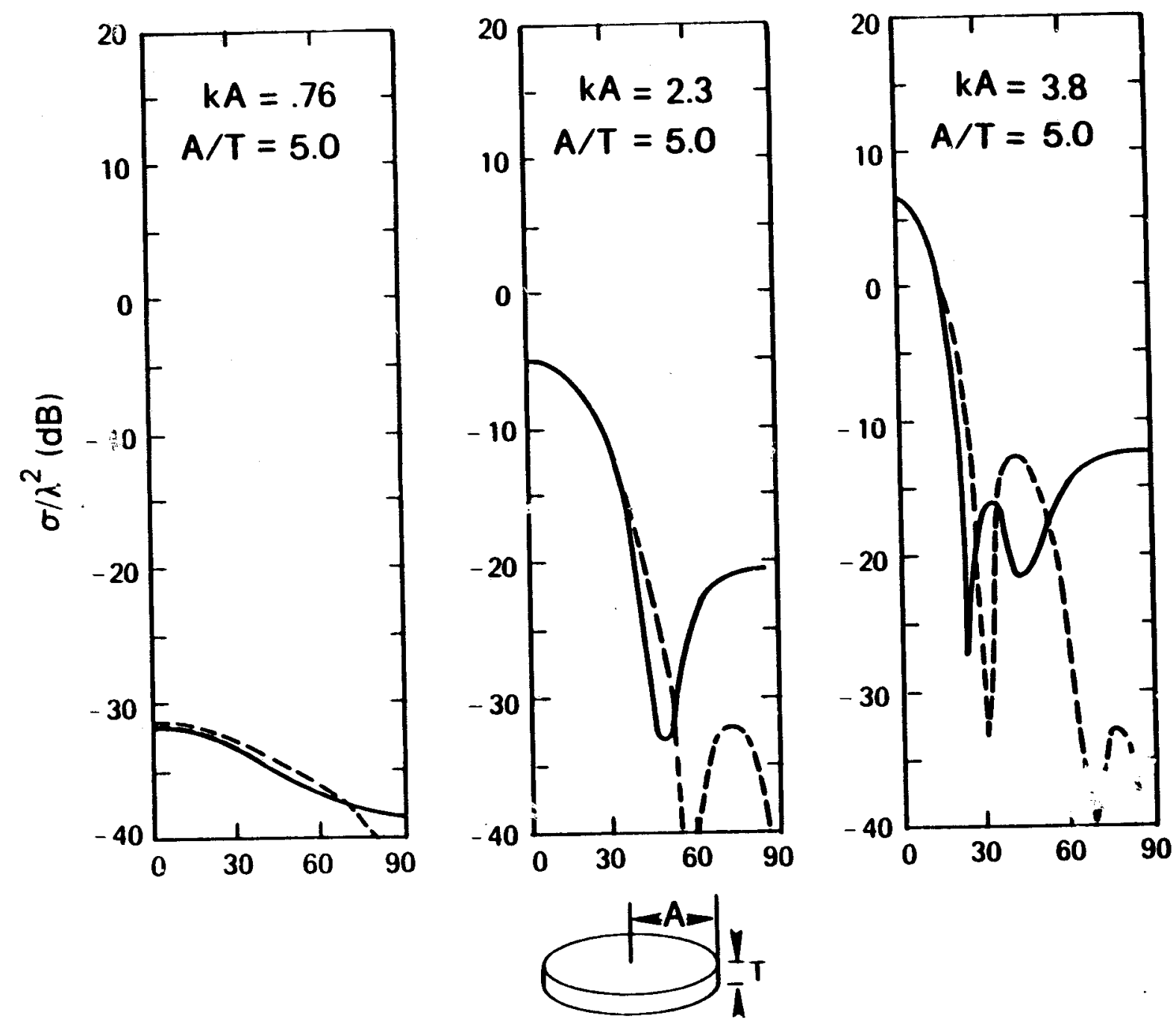

Figure 5. Scattering from a circular dielectric disk. The measurements (solid lines) are from Allan \& McCormick (1980). 

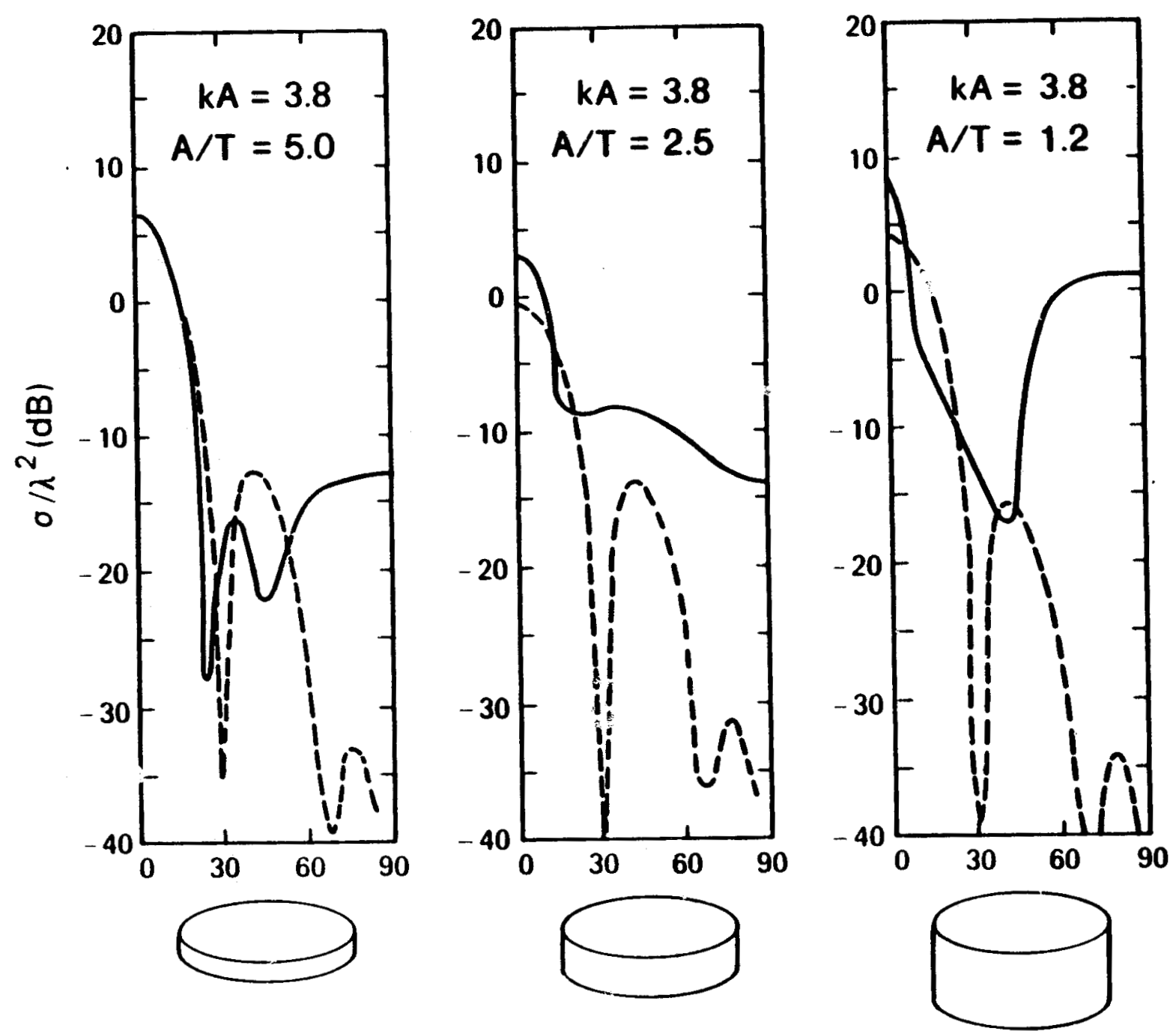

Figure 6. Scaltering from circular dielectric disks with $k A=3.8$ and different thickness. The measurements (solid curves) are from Allan \& McCormick (1980). 

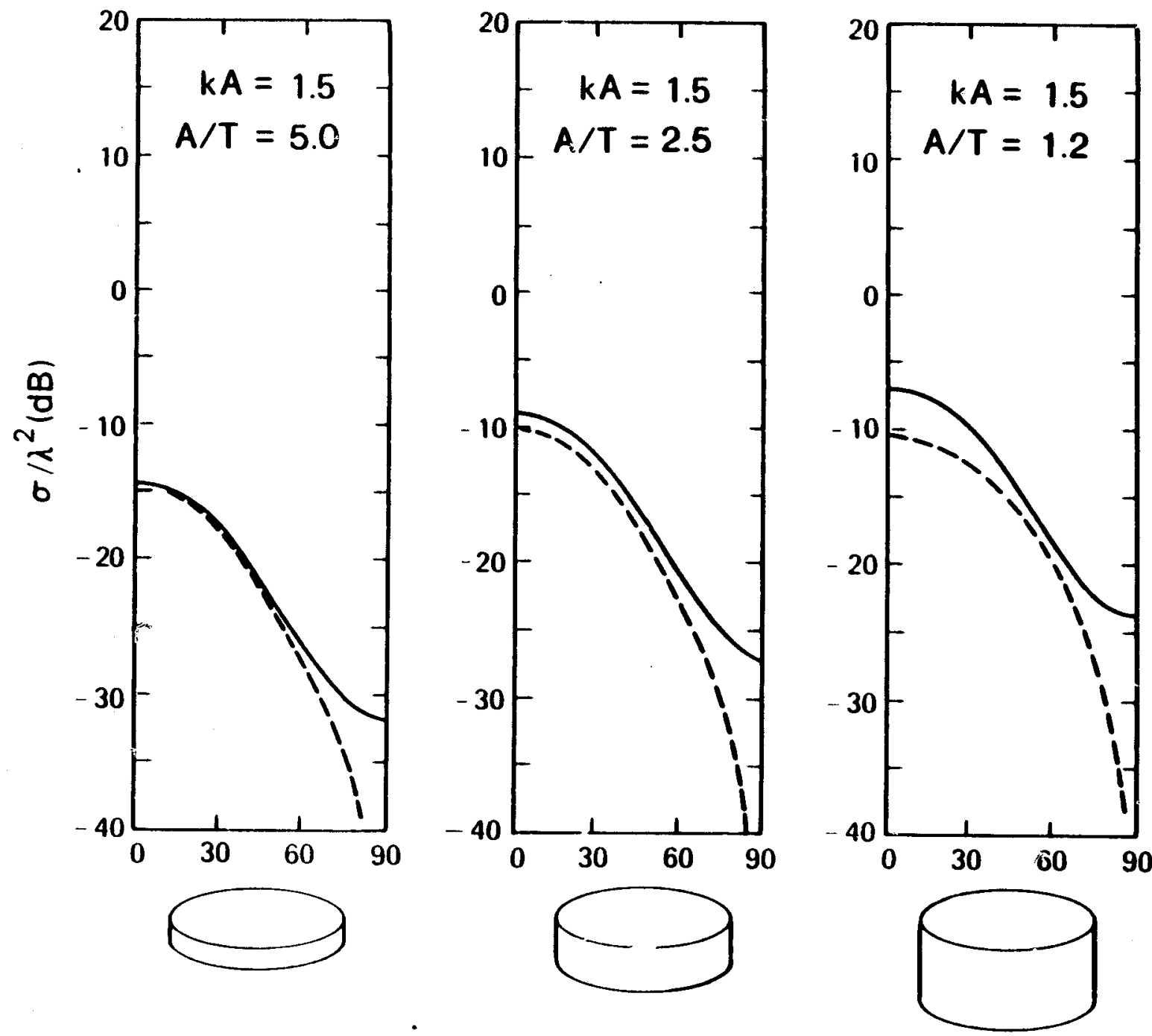

Figure 7. Scattering from circular dielectric disks with $k A=1.5$ and different thickness. The data (solid lines) are from Allan \& McCormick (1980). 